\title{
Low complexity symbol detection method for multilevel 2-D optical storage based on a linear channel model
}

\author{
Abdolhosein Moinian \\ Lina Fagoonee \\ Bahram Honary \\ Lancaster University \\ Department of Communication Systems \\ Lancaster LA1 4YR, United Kingdom \\ E-mail: a.moinian@lancaster.ac.uk
}

\begin{abstract}
A symbol detection scheme based on the Viterbi algorithm that simultaneously processes subsets of 2-D data in the presence of Gaussian noise was recently proposed for binary 2-D optical storage (TwoDOS). In the case of multilevel TwoDOS, a straightforward fullfledged maximum likelihood symbol detector, or even the previous Viterbi-based algorithm, is not an ideal solution due to complexity restrictions. We propose a suboptimum low complexity symbol detector, which still performs within the accepted performance bound for optical storage. We describe the procedures involved in designing and developing a practical symbol detection scheme for multilevel TwoDOS by analyzing the signal values generated by a linear channel model in the presence of Gaussian noise. Our proposed detection scheme exploits the properties of the 2-D data format on the disk, and is flexible enough to accommodate performance and complexity restrictions for optical storage applications. () 2005 Society of Photo-Optical Instrumentation Engineers.
\end{abstract} [DOI: 10.1117/1.2087647]

Subject terms: detection; optical storage; detector.

Paper 040915R received Dec. 1, 2004; revised manuscript received Mar. 17, 2005; accepted for publication Apr. 5, 2005; published online Oct. 18, 2005.

\section{Introduction}

A binary 2-D optical storage ${ }^{1}$ (TwoDOS) disk has been developed to increase capacity and data rate with factors of up to 2 and 10, respectively, over "blu-ray disk" (BD, formerly known as DVR) technology ${ }^{2}$ by developing a new technique of storing data in a 2-D format. Using multilevel techniques over binary TwoDOS, an even higher increase in capacity and data rate can be realized. TwoDOS is based on a so-called broad spiral, along which information is written as a limited number of parallel data rows stacked on each other and arranged in a coherent 2-D format, with no spacing in between the rows. A guard band consisting of one row of known land symbols is located between every two successive revolutions of the broad spiral. The horizontal parallel data rows in the broad spiral are arranged in a honeycomb format using hexagonal lattices. The motivation behind the hexagonal structure ${ }^{1}$ is that it provides a $15 \%$ higher packing rate than the ordinary square lattice format. ${ }^{3,4}$ The lattice parameter $a_{H}$ is the distance between the centers of two neighboring lattice cells.

Multilevel TwoDOS is characterized by $M$ level symbols, consisting of 1 land symbol, which is the flat reflecting surface, and $M-1$ pit symbols, where $M>2$. Symbols are represented by $0,1, \ldots M-1$, where 0 represents a land symbol, 1 is the pit symbol with the smallest pit area, and the remaining symbols are arranged in ascending order with $M-1$ representing the pit symbol with the largest area. The pit symbols are mastered as pit holes, each with a unique pit radius, centered within the hexagonal cell that is avail-

0091-3286/2005/\$22.00 @ 2005 SPIE able for each symbol. All pits have a fixed phase depth $\Phi$. The reflected light traveling to the bottom of a pit and back out produces a phase depth of $\Phi=2 \pi\left(2 d / \lambda^{\prime}\right)$ with respect to light reflected from the all-land area. $\lambda^{\prime}$ is the wavelength of the laser light inside the cover layer. For simplicity, the phase depth is chosen to be $\Phi=\pi$, which results in $d=\lambda^{\prime} / 4$.

In this work, we consider multilevel modulation with four symbols: one land and three pits, $P_{1}, P_{2}$, and $P_{3}$, as illustrated in Fig. 1(a). The pit radii are chosen such that their areas are linear in the ratio 1:2:3, and the maximum pit area covers $50 \%$ of the total hexagonal cell area to avoid signal folding. ${ }^{5}$ We consider 11 parallel rows in the broad spiral and a lattice parameter $a_{H}=182 \mathrm{~nm}$. The readout channel is simulated in the form of a linear channel model, which has a 2-D low-pass filter characteristic and is based on the Braat-Hopkin's formula. ${ }^{6,7}$ This model has been extended to a 2-D function for binary ${ }^{6}$ and multilevel TwoDOS. ${ }^{8}$ The readout channel is characterized by inter symbol interference (ISI) and Gaussian noise. The signalprocessing path from photodetector signals to detected symbols comprises a cascade of signal processing blocks similar to that proposed for binary TwoDOS. ${ }^{9}$

The work is structured as follows. Section 2 describes the linear channel model. In Sec. 3, signal patterns generated by the channel model are described, the summationgrouping method is presented as an efficient way of grouping identical types of signal values to simplify the process of symbol detection, and we summarize results of a statistical analysis of the signal values to determine thresholds for the initialization of our symbol detection scheme. The proposed low complexity symbol detection scheme is pre- 


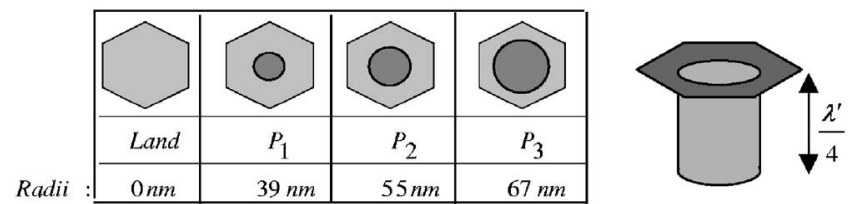

Fig. 1 Four-level TwoDOS symbols within a hexagonal cell and relative pit radii: (a) surface view and (b) section view.

sented in Sec. 4. The scheme is validated by simulation results in Sec. 5, and we show that we achieve the performance expected for optical storage applications. ${ }^{10,11}$ Conclusions of this study are given in Sec. 6 .

\section{Linear Channel Model}

The linear channel model is simple from a signal processing point of view, because it is less complex in comparison with nonlinear signal processing models such as the vector diffraction model ${ }^{12}$ or the scalar diffraction model ${ }^{13}$ However, the linear model does not take into account nonlinear effects such as pit asymmetry. We make the following valid assumptions: 1. linear ISI has a relatively higher influence than nonlinear contributions, and 2. a precompensation iteration process ${ }^{7}$ located in the write channel is able to remove the effect of nonlinear ISI.

Traditionally, in 1-D optical storage (CD, DVD, and $\mathrm{BD})$, readout channels are often simulated by a linear model, ${ }^{14}$ which is characterized by its modulation transfer function (MTF) as derived in the Braat-Hopkin's formula. ${ }^{6}$ For the purpose of TwoDOS, the formalism is extended to the 2-D character, ${ }^{5,8}$ as expressed in Eq. (1). The MTF has a low-pass behavior with a hard cutoff frequency beyond which no information is transferred and is given by $v_{c}$ $=2 N A / \lambda$. Parameter $\lambda$ is the wavelength of the laser beam and $N A$ is the numerical aperture of the objective lens in the optical pick-up unit. These parameters are chosen to be the same as for BD specifications, ${ }^{2}$ i.e., $\lambda=405 \mathrm{~nm}$ and $N A$ $=0.85$. Parameter $v$ is the spatial frequency of the information on the disk and $\theta$ is the azimuth in the 2-D spatial frequency plane. The 2-D MTF is shown in Fig. 2, where $v_{c}$ is normalized to 1 . The amplitude of the 2-D MTF function decreases monotonically from dc to the cutoff frequency and is circularly symmetric around the amplitude axis, which is similar to a 2-D cone with radius corresponding to the $v_{c}$.

$$
\begin{aligned}
& H_{2 \mathrm{D}}(v, \theta) \\
& =\left(\begin{array}{rl}
\frac{2}{\pi}\left\{\arccos \left(\frac{v}{v_{c}}\right)-\frac{v}{v_{c}}\left[1-\left(\frac{v}{v_{c}}\right)^{2}\right]^{1 / 2}\right\} & \begin{array}{l}
v \\
0
\end{array} \\
v & >v_{c}
\end{array}\right.
\end{aligned}
$$

$0 \leqslant \theta \leqslant 2 \pi$.

In a 2-D lattice, each symbol $S$ is uniquely identified by its position $j$ in shell $i$. The symbol value $S_{i, j}$ is assigned the value $0,1,2$, and 3 for land, $P_{1}, P_{2}$, and $P_{3}$, respectively. These symbol values $S_{i, j}$ are modulated to bipolar values, using generic Eq. (2), as input to the linear channel model for any value of $M \geqslant 2$, which is equivalent to at least one pit.

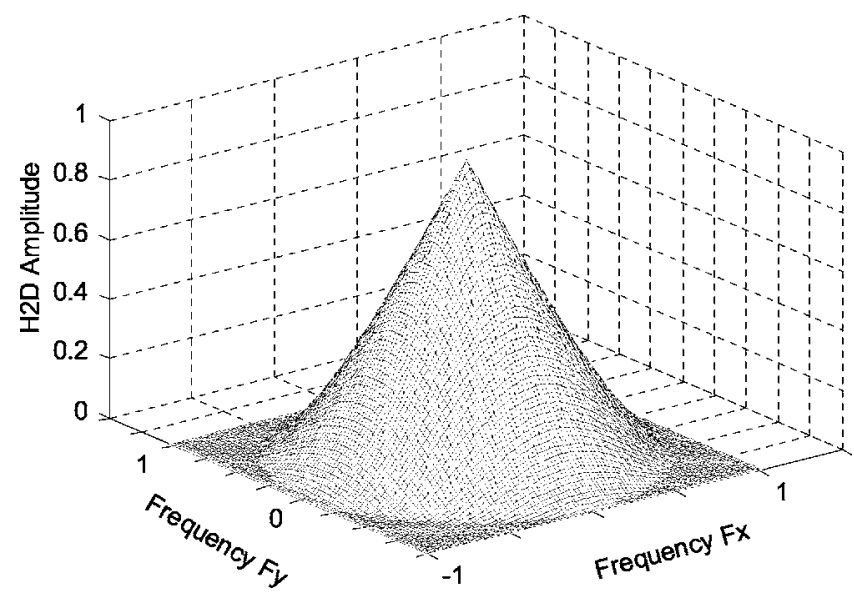

Fig. 2 2-D MTF.

$S_{i, j}^{b}=M-1-2 S_{i, j}$.

For $M=4$, we assume the areas of pit symbols are linearly related in the ratio $1: 2: 3$, and the amplitude of the signal waveform diffracted from a pit is linearly dependent on the area of the pit. The highest $S_{i, j}^{b}$ value is associated with the land case, where almost (taking residual ISI into account) all light is reflected from the plane of information on the disk. Each pit symbol is assigned a value indicative of its relative pit area.

The symbol-synchronous signal waveform $I_{i, j}$ is obtained as the linear convolution of the bipolar symbol value $S_{i, j}^{b}$ and the impulse response function (IRF) of the channel, denoted by $w_{i}$, also referred to as "tap values." The tap values are computed as the Fourier transform of the MTF ${ }^{13}$ given by Eq. (1). Each shell is assigned one tap value because all symbols in that shell have the same distance from the center due to the symmetry of the hexagonal lattice. The calculation $I_{i, j}$, is shown in Eq. (3), where $N_{s}$ is the total number of shells that lie inside the laser spot; $N_{i}$ denotes the number of cells in the $i$ 'th shell.

$I_{i, j}=\sum_{i=0}^{N_{s}}\left\{w_{i} \times \sum_{j=1}^{N_{i}} S_{i, j}^{b}\right\}$.

In Table 1, the normalized tap values (with respect to the central tap value) and their distances from the center as a factor of $a_{H}$ are shown. As expected, the first shell has the maximum tap value. However, unlike the linear trend in the MTF, the tap values do not decrease monotonously as the distance, which is a factor of lattice parameter $a_{H}$ $=182 \mathrm{~nm}$, from the center increases. This phenomenon is explained by the airy characteristic ${ }^{15}$ of the Fourier transform of the MTF.

\section{Signal Patterns}

\subsection{Readout Data Patterns}

During readout, each laser spot is centered on one cell but may also cover a number of neighboring cells due to the relatively small size of the cell with respect to the laser spot. This implies that when the spot scans a symbol, part of the reflected light is formed by diffraction of the light 
Table 1 The normalized tap values and their distances from the center.

\begin{tabular}{ccc}
\hline \hline$i$ & Distance $\left(a_{H}\right)$ & $w_{i}$ \\
\hline 0 & 0.00 & 1.00000 \\
1 & 1.00 & 0.27005 \\
2 & 1.73 & -0.00159 \\
3 & 2.00 & -0.00323 \\
4 & 2.65 & 0.01068 \\
5 & 3.00 & 0.00672 \\
6 & 3.46 & -0.00356 \\
7 & 3.60 & -0.00086 \\
8 & 4.00 & 0.00267 \\
\hline \hline
\end{tabular}

from neighbor cells. This is the cause of ISI in TwoDOS. By increasing the data density, i.e., choosing smaller $a_{H}$, more cells lie within the laser spot, which in turn results in a higher ISI.

The distance of the neighbors from the central cell affects the contribution of ISI from each neighbor. These neighbors can be grouped in shells, where each shell consists of a number of cells all with an identical distance from the central cell. As illustrated in Fig. 3, symbols in a shell are indexed with respect to their distance from the center labeled 0; nearest neighbors indexed 1 belong to the first shell, symbols in the second shell are indexed with 2, and so on. The central symbol and its six nearest neighbors in the first shell form a cluster.

\subsection{Signal Levels}

Tap values are a measure of ISI contribution from each shell surrounding the central symbol. As shown in Table 1, symbols in the first shell are the dominant source of ISI, being closest to the central symbol. The signal values for the proposed medium, using the model just described, are

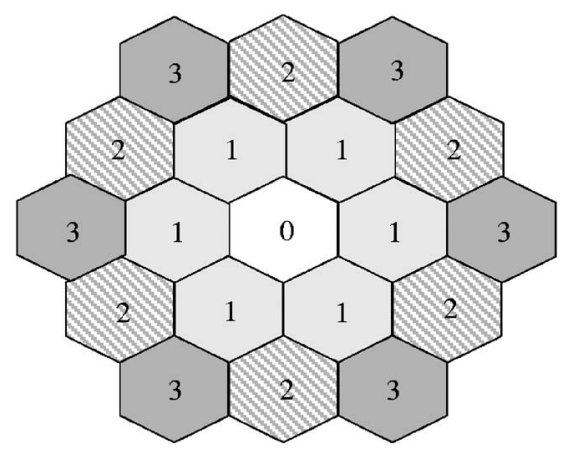

Fig. 3 Distribution of hexagonal cells in shells around the central symbol.

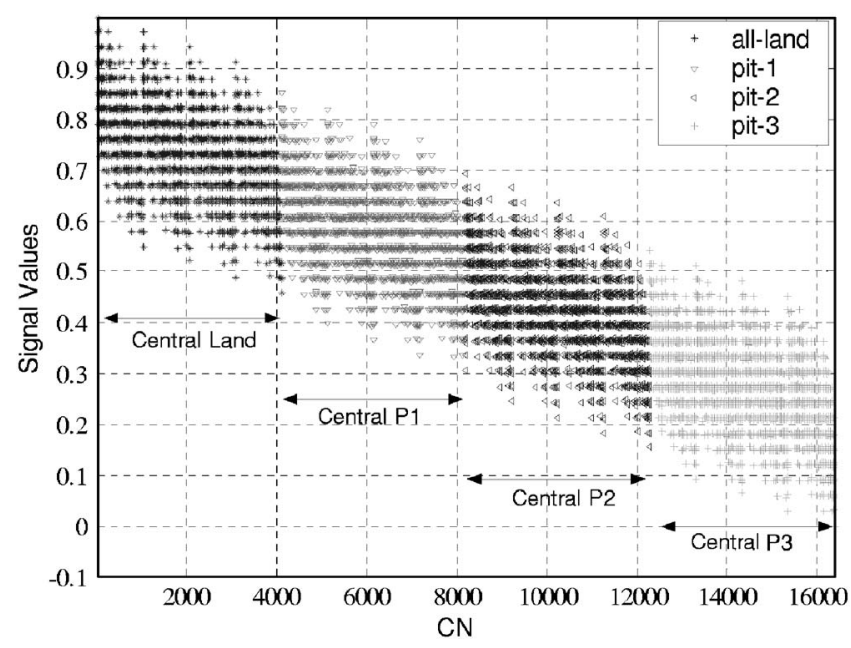

Fig. 4 Signal levels for all 16,384 cluster permutations.

identified by a plot of signal values for the complete set of all possible clusters. The total number of cluster permutations $N_{T}$ is $4^{7}$ or 16,384 .

The indexing for signal values is based on the number and position of pits in the first shell. The normalized signal values for the 16,384 permutations are shown in Fig. 4. Indexing is given as a function of the cluster indices $(C N)$ :

$C N=4^{6} \cdot S_{0,0}+\sum_{j=1}^{6}\left[S_{1, j} \cdot 4^{(j-1)}\right]$.

There are four distinct sections: cluster numbers 1 to 4096 represent clusters with a common central land symbol; and cluster numbers 4097 to 8193 represent clusters with a common central symbol $P_{1}$, etc. In each section, indexing starts with the cluster with the least pit area (all-land cluster) and increases with pit area until the largest pit area with seven $P_{3}$ 's in the cluster is reached.

\subsection{Summation Grouping}

The area of neighboring pits determines the amount of linear interference. As established before, the first shell is considered to be the dominant source of ISI. By choosing the radii of the pits, such that their respective areas become linearly related, for a fixed central symbol, all clusters with the same overall pit area in the first shell will have almost similar signal values, so they can be grouped together. The mean of signal values of each group is referred to as a signal level.

Definition 1. Let $N p_{3}, N p_{2}$, and $N p_{1}$ denote the number of pits $P_{3}, P_{2}$, and $P_{1}$, respectively, in the first shell. Group $G_{S_{0,0}, j}$ is a summation group, where all clusters with symbol $S_{0,0}$ in the center are related as follows:

$3 \cdot N_{p_{3}}+2 \cdot N_{p_{2}}+1 \cdot N_{p_{1}}=j$ for $0 \leqslant j<19$.

The 16,384 clusters can be grouped according to Definition 1, yielding 19 signal levels per section, as shown in Fig. 5. The indexing in each section starts with the smallest pit area in the first shell $(j=0$; six lands) and ends with the largest pit area in the first shell $\left(j=18\right.$; six $P_{3}$ 's). For ex- 


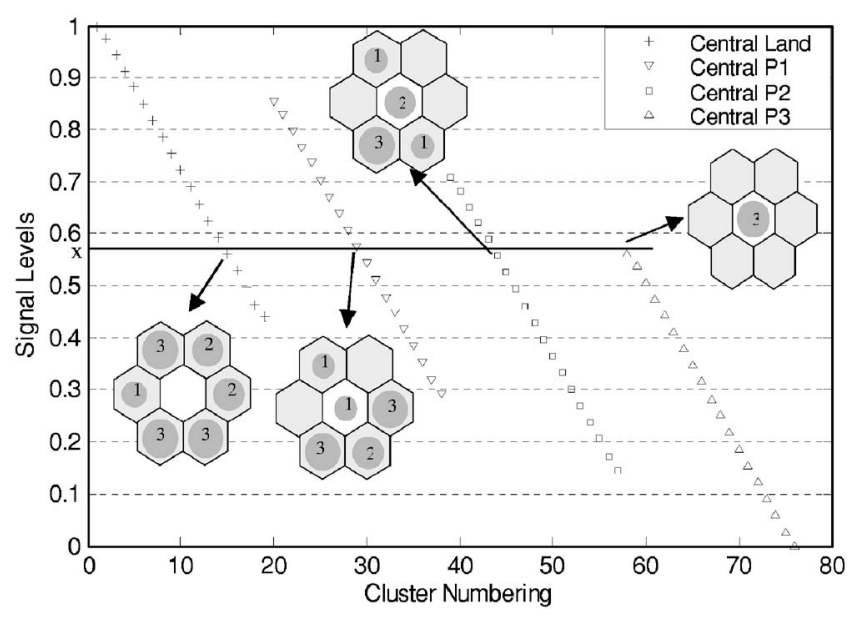

Fig. 5 Signal levels after summation grouping, showing four possible clusters with the same signal level of 0.57 .

ample, three members of $G_{0,3}$ with $S_{0,0}=0$ could have in the first shell: three adjacent $P_{1}$ and three land symbols, three $P_{1}$ symbols each separated with a land symbol, or one $P_{3}$ symbol and five land symbols.

\subsection{Probability Distribution}

In general each signal value varies due to the ISI from the outer shells (second shell, third shell, etc.) and additive white Gaussian noise (AWGN), which is introduced by the optical channel, as shown in the system model in Fig. 6. In the presence of $\mathrm{AWGN}$ with $\mathrm{SNR}=25 \mathrm{db}$, and simulating ISI contributions from two outer shells, the overall probability distribution for four-level TwoDOS is shown in Fig. 7. Three threshold levels, $T_{1}, T_{2}$, and $T_{3}$, are used in the symbol detection algorithm to initialize symbol values, e.g., the most likely symbol for the normalized signal values between 0 and 0.3 is land, and in the case of normalized signal values between 0.3 and 0.5 , the most likely symbol is $P_{1}$, etc.

\section{Symbol Detection}

To determine a suitable symbol detection scheme for the multilevel TwoDOS linear channel model, we address the complexity and performance tradeoffs. Considering a broad spiral with $N_{r}$ rows for the four-level TwoDOS, a straightforward full-fledged Viterbi symbol detector requires $4^{(k-1) N r}$ number of states, where $k$ is the number of symbols in the tangential direction per state in the Viterbi detector. As an example, for the model that we considered in this work (one central tap and six nearest neighbor taps), we have that $k=3$. Thus, when using a meta-spiral with $N_{r}$

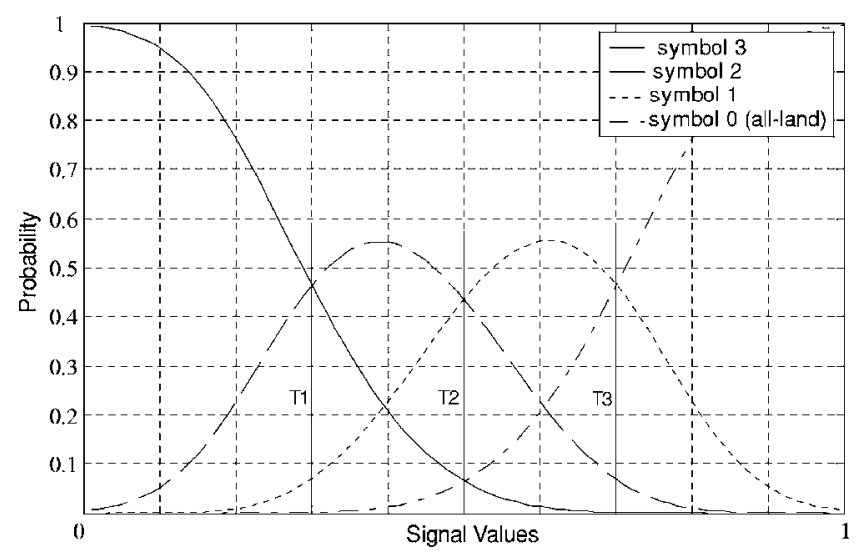

Fig. 7 Probability distribution of signal values.

$=11$, there will be $4^{22}$ states in the Viterbi trellis. This is clearly impractical from a hardware point of view. To reduce the complexity of the maximum likelihood detection scheme, Viterbi-based algorithms that simultaneously process multiple rows from a set of 2-D data have been proposed, ${ }^{16,17}$ and the complex problem of performing symbol detection over a meta-spiral is broken down into a number of bit detectors, each processing along a set of adjacent tracks. Similar work was developed for binary TwoDOS as a stripe wise Viterbi detector (SWD). ${ }^{18}$ However the complexity of the SWD for multilevel TwoDOS has prompted us to suggest a less complex scheme that exploits the properties of the multilevel 2-D readout channel.

The proposed detection method compares the channel readout with the signal levels obtained by summation grouping of the linear model output. If two clusters with different central symbol values have similar signal values, there will be a difference between the sum of their nearest neighbors. In other words, the central symbol value can be detected based on its signal value and estimating the sum of its nearest neighbors to determine the closest cluster match, which is addressed later. An example was illustrated in Fig. 5 for the normalized signal value of 0.57 , which could correspond to clusters from groups $G_{0,14}, G_{1,9}, G_{2,5}$, and $G_{3,0}$.

\subsection{Sliding Window Patterns and Corresponding Detection Algorithms}

The sliding window pattern consists of a limited number of adjacent cells grouped to include a number of central symbols surrounded by their nearest neighbors, as shown in Fig. 8. In the detection scheme, the central symbol(s) is

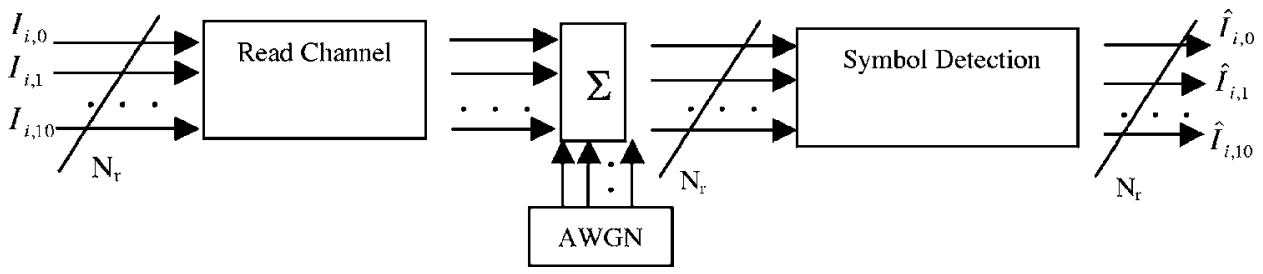

Fig. 6 The block diagram of the overall system. 


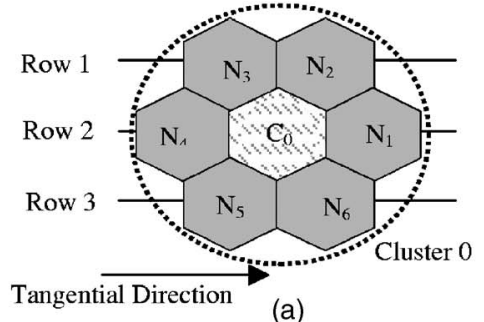

(a)

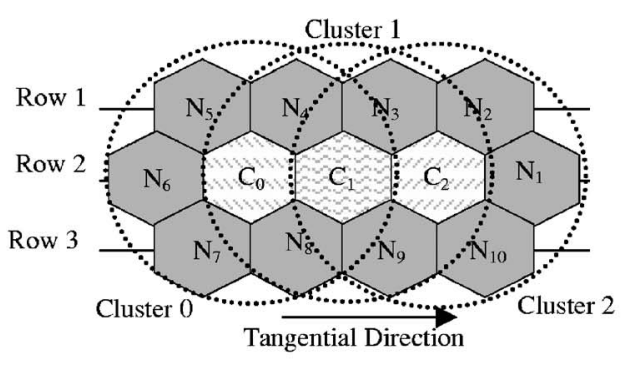

(c)

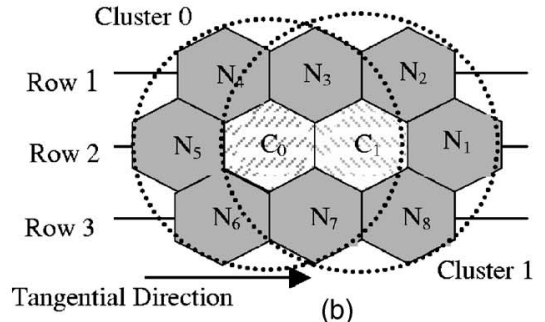

(b)

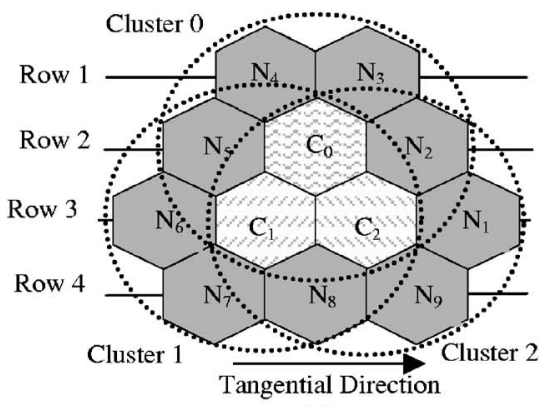

(d)

Fig. 8 Sliding window patterns: (a) pattern $A$, (b) pattern $B$, (c) pattern $C$, and (d) pattern $D$.

(are) updated according to the sum of their nearest neighbors. Four different window patterns are proposed. In Fig. 8(a), pattern $A$ consists of a cluster; in Fig. 8(b), pattern $B$ consists of two central symbols surrounded by eight nearest neighbors; in pattern $C$, as shown in Fig. 8(c), there are three central symbols and ten nearest neighbors, whereas pattern $D$, as shown in Fig. 8(d), consists of three central symbols and nine nearest neighbors. For patterns $A, B$, and $C$, symbol detection can be performed by shifting the sliding window pattern along three adjacent data rows in the tangential direction and updating the central symbol value(s) via the detection algorithm, which is explained in the next section. Pattern $D$ is spread over four rows, and the symbols in the two middle rows are updated.

We refer to the block performing symbol detection for a number of rows within the sliding window pattern as a detector. With $N_{r}=11$, as shown in Fig. 9(a), ten detectors $D_{1}, D_{2}, \ldots D_{10}$, using one of the patterns $A, B$, or $C$, operate on the broad spiral from the outer rows toward the middle row of the spiral. The input to each detector consists of the signal values on three adjacent rows. The output sequence consists of an updated estimate for the symbol values in the middle row, i.e., row 2. Out $t_{i}$ represents the output for each detector $D_{i}$. The two outer detectors $D_{1}$ and $D_{2}$ use the known symbols in the guard band, and together with the other two input rows per detector, provide an updated estimate for their output rows $\mathrm{Out}_{1}$ and $\mathrm{Out}_{2}$ respectively. $\mathrm{Out}_{1}$ and $\mathrm{Out}_{2}$ are used as extrinsic information for the next set of detectors, $D_{3}$ and $D_{4}$, to provide the next set of output rows, $\mathrm{Out}_{3}$ and $\mathrm{Out}_{4}$. The procedure is repeated for the rest of the detectors.

The detection algorithm for pattern $D$ is slightly different because the pattern is spread over four rows. Each detector $D_{i}$ receives symbol values from four rows as input and provides the estimated information for the two middle rows, rows 2 and 3, as an output sequence. Two different configurations are illustrated in Figs. 9(b) and 9(c). The first configuration uses ten detectors, which are shifted by one row toward the middle of the spiral. As a result, only one of the outputs of each detector provides the final estimated output per iteration, i.e., $E_{i}$ from detector $D_{i}$ is overwritten by $O u t_{i+2}$ of the next detector, $D_{i+2}$, as shown in Fig. 9(b). In the second configuration, detectors are shifted by two rows toward the middle of the broad spiral, and both outputs are considered as the final estimated output per iteration, as in Fig. 9(c). In the last detector, because of the odd number of rows, Out $_{6}$ overwrites $E_{6}$. The combination of the detectors forms a V-shaped detection algorithm (VDA) along the broad spiral.

\subsection{Symbol Detection}

As the iterative nature of the symbol detection algorithm suggests, each VDA can operate one after the other along the direction of the broad spiral, and each of them forms an iteration of the total symbol detection process.

Prior to the first iteration, all cells are initialized with the highest probable symbol value by using threshold detection, based on the probability of each central symbol as explained in Sec. 3. Four reference values, $R f_{S_{0,0}}$, are defined per cell. For each central symbol $S_{0,0}, R f_{S_{0,0}}$ is the sum of the nearest neighbors in clusters of the relevant summation groups. Using the example of Fig. 5, based on the normalized signal value of 0.57 , the reference levels are $R f_{0}=14, R f_{1}=9, R f_{2}=5$, and $R f_{3}=0$. If no summation group was available for a certain symbol, the detector returns an out-of-range flag such as -1 .

For pattern type A, each of the 11 detectors updates its central symbol and shifts one cell along the detection process. To update the central symbol, four distances $d_{0}^{l}, d_{1}^{l}, d_{2}^{l}$, and $d_{3}^{l}$ are calculated as in Eq. (7). At the $l$ 'th iteration, $S_{C_{0}}$ is the sum of the six nearest neighbors $\left(N_{1} \ldots N_{6}\right)$, and $C_{0}$ is 


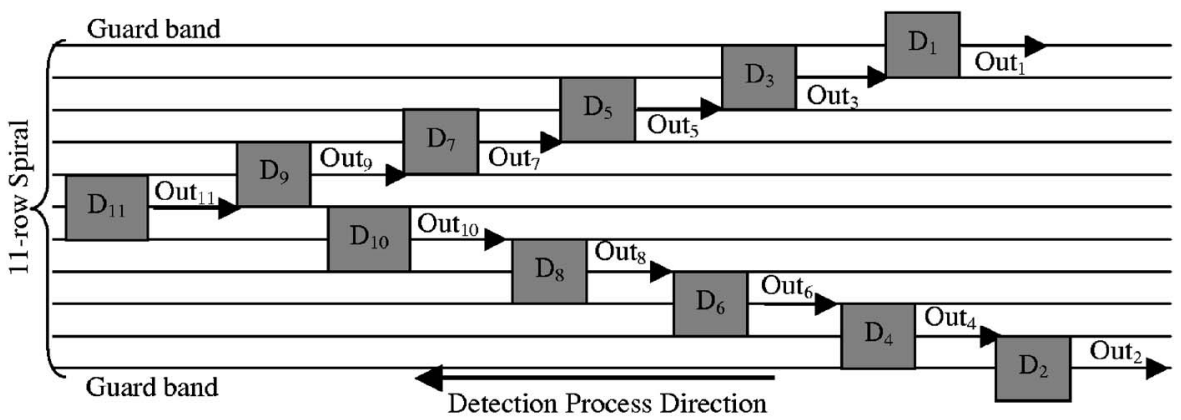

(a)

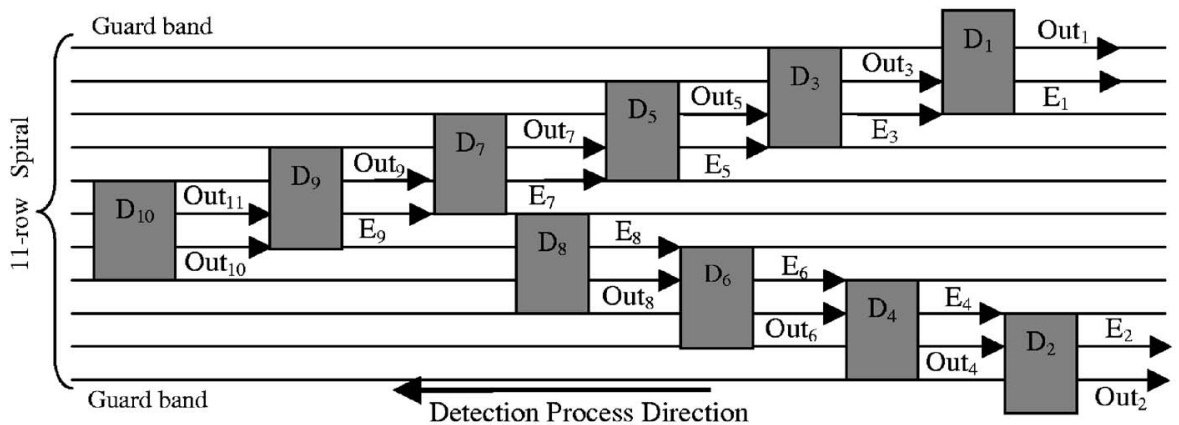

(b)

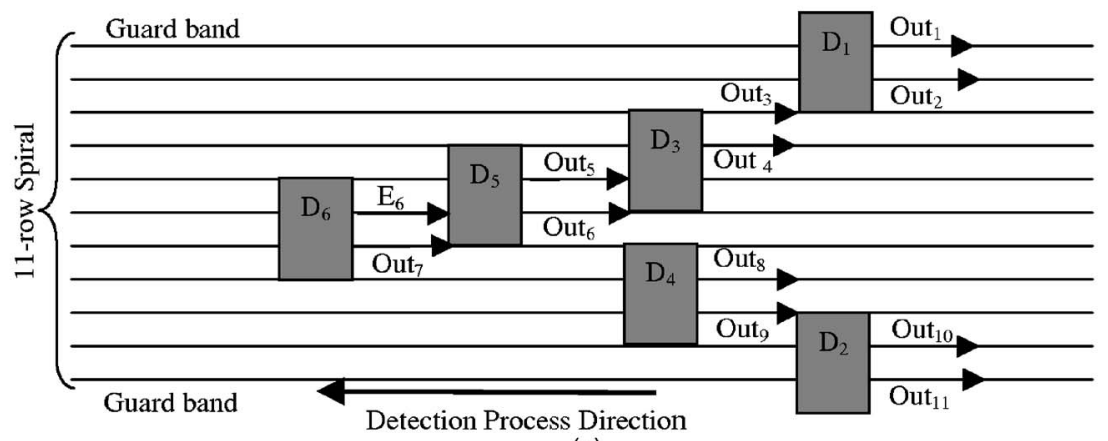

(c)

Fig. 9 (a) VDA for patterns $A, B$, and $C$. (b) Ten-detector VDA for pattern $D$. (c) Six-detector VDA for pattern $D$.

the central cell symbol value. The central symbol is updated with the symbol value, which results in the smallest $d_{C_{0}}^{l}$

$d_{C_{0}}^{l}=\left(S_{C_{0}}-R f_{C_{0}}\right)^{2}$ for $C_{0}=0,1,2,3$.

For pattern type $B$, considering all different combinations of $C_{0}$ and $C_{1}$, two sets of distances are calculated, $d_{\text {cluster } 0}^{l}\left(C_{0}, C_{1}\right)$ and $d_{\text {cluster } 1}^{l}\left(C_{0}, C_{1}\right)$ for $0 \leqslant C_{0}, C_{1} \leqslant 3$, where each set consists of 16 distances due to all possible combinations of $C_{0}$ and $C_{1}$.

$d_{\text {cluster } 0}^{l}\left(C_{0}, C_{1}\right)=\left(S_{C_{0}}-R f_{C_{0}}\right)^{2}$ for $0 \leqslant C_{0}, C_{1} \leqslant 3$,

$d_{\text {cluster } 1}^{l}\left(C_{0}, C_{1}\right)=\left(S_{C_{1}}-R f_{C_{1}}\right)^{2}$ for $0 \leqslant C_{0}, C_{1} \leqslant 3$.

$S_{C_{0}}$ is the sum of the symbol values of the nearest neighbors in cluster $0\left(N_{3} \ldots N_{7}\right.$ and $\left.C_{1}\right) . S_{C_{1}}$ is the sum of the symbol values of the nearest neighbors in cluster $1\left(N_{1} \ldots N_{3}, N_{7}\right.$, $N_{8}$, and $\left.C_{0}\right) . S_{C_{0}}$ and $S_{C_{1}}$ are both dependent on the symbol values of $C_{0}$ and $C_{1}$. Central symbol values $C_{0}$ and $C_{1}$ are updated by the respective $D_{\min }^{B}\left(C_{0}, C_{1}\right)$, as shown in Eq. (9):

$$
\begin{aligned}
D_{\min }^{B}\left(C_{0}, C_{1}\right)= & \min \left[d_{\text {cluster } 1}^{l}\left(C_{0}, C_{1}\right)\right. \\
& \left.+d_{\text {cluster } 0}^{l}\left(C_{0}, C_{1}\right)\right] \text { for } 0 \leqslant C_{0}, C_{1} \leqslant 3 .
\end{aligned}
$$

After each central symbol update, the window shifts one or two cells in the tangential direction, and the detector updates the symbols of the entire row. In this work, the results for one shift are presented.

In the case of pattern types $C$ and $D$, three central symbol values, $C_{0}, C_{1}$, and $C_{2}$ are updated. Each symbol is respectively located in the center of cluster 0 , cluster 1 , and cluster 2, respectively, as shown in Figs. 8(c) and 8(d). Therefore, three sets of distances, $d_{\text {cluster } 0}^{l}\left(C_{0}, C_{1}, C_{2}\right)$, 
$d_{\text {cluster } 1}^{l}\left(C_{0}, C_{1}, C_{2}\right)$, and $d_{\text {cluster } 2}^{l}\left(C_{0}, C_{1}, C_{2}\right)$ are introduced for $0 \leqslant C_{0}, C_{1}, C_{2} \leqslant 3$, where each set consists of 64 distances due to all possible combinations of $C_{0}, C_{1}$, and $C_{2}$.

$d_{\text {cluster } 0}^{l}\left(C_{0}, C_{1}, C_{2}\right)=\left(S_{C_{0}}-R f_{C_{0}}\right)^{2}$ for $0 \leqslant C_{0}, C_{1}, C_{2} \leqslant 3$,

$d_{\text {cluster } 1}^{l}\left(C_{0}, C_{1}, C_{2}\right)=\left(S_{C_{1}}-R f_{C_{1}}\right)^{2}$ for $0 \leqslant C_{0}, C_{1}, C_{2} \leqslant 3$,

$d_{\text {cluster } 2}^{l}\left(C_{0}, C_{1}, C_{2}\right)=\left(S_{C_{2}}-R f_{C_{2}}\right)^{2}$ for $0 \leqslant C_{0}, C_{1}, C_{2} \leqslant 3$.

$S_{C_{0}}, S_{C_{1}}$, and $S_{C_{2}}$ are the sum of the symbol values of the nearest neighbors in cluster 0 , cluster 1 , and cluster 2, respectively, and they are all dependant on the symbol values of $C_{0}, C_{1}$, and $C_{2}$, respectively. Central symbol values $C_{0}$, $C_{1}$, and $C_{2}$ are updated by the respective $D_{\min }^{C, D}\left(C_{0}, C_{1}, C_{2}\right)$, as shown in Eq. (13):

$$
\begin{aligned}
D_{\min }^{C, D}\left(C_{0}, C_{1}, C_{2}\right)= & \min \left[d_{\text {cluster } 1}^{l}\left(C_{0}, C_{1}, C_{2}\right)\right. \\
& +d_{\text {cluster } 0}^{l}\left(C_{0}, C_{1}, C_{2}\right) \\
& \left.+d_{\text {cluster } 2}^{l}\left(C_{0}, C_{1}, C_{2}\right)\right] \\
& \text { for } 0 \leqslant C_{0}, C_{1}, C_{2} \leqslant 3 .
\end{aligned}
$$

After the central symbol update, the sliding window shifts one cell in the direction of the detection process, and the updating process is repeated.

\section{Simulation Results}

Typically, the limit of the error correction code in optical storage applications is about $4 \times 10^{-3}$ byte error rate (BER), as in the BD disk. ${ }^{19}$ For random errors with independent symbol error events in a byte, the BER corresponds to a bit error rate (bER) of $5 \times 10^{-4}$. This is because an error correction decoder is assumed to correct the remaining errors at the output of the symbol detector.

In Fig. 10, the relative performance of the four types of sliding window patterns in the presence of AWGN is shown. Preliminary results from the experimental TwoDOS device for binary TwoDOS suggest an operation point with a signal-to-noise ratio (SNR) of $32 \mathrm{~dB}$. At this operation point, patterns $A, B, C$, and $D$ (with ten detectors), provide bERs of $7.6 \times 10^{-4}, 3.1 \times 10^{-4}, 2.7 \times 10^{-4}$, and $2 \times 10^{-4}$, respectively, using seven iterations for the detection process.

The relative performance of the first three configurations is indicative of the number of extrinsic symbol probabilities being considered, where pattern $A$ with only six extrinsic symbols has the worst performance, and pattern $C$ has ten extrinsic symbols. Owing to its 2-D sliding window configuration, where the distance between extrinsic and central symbols is smaller, pattern $D$ outperforms pattern $C$, although it has only nine extrinsic symbols. Pattern $D$ with ten detectors outperforms the same pattern with six detectors, because more extrinsic information is generated by VDAs shifting by one row as opposed to two rows for the latter case. Any of these four patterns can be used depend-

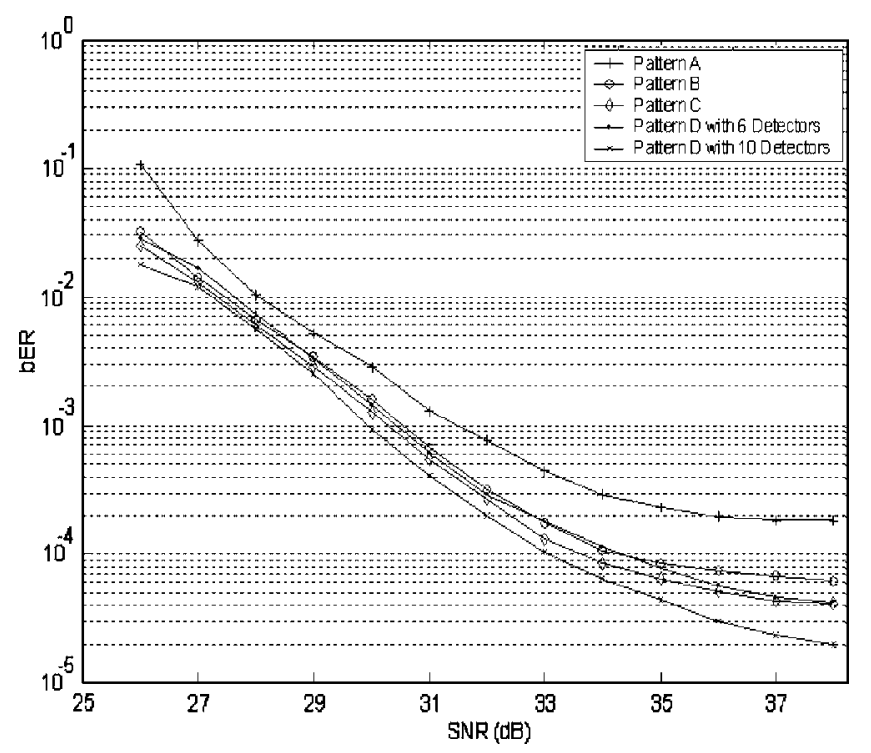

Fig. 10 bER performance for all window patterns (seven iterations).

ing on the processing complexity and memory tradeoffs with respect to performance.

In Fig. 11, the performances for up to seven iterations over AWGN of SNR $=32 \mathrm{~dB}$ are represented. After five iterations, the performance curve saturates to an error floor, and further detection iterations do not significantly improve the performance. Based on the MTF characteristics, the ISI contribution to the signal values measured at the central symbol is most significant from cells in the first shell. Referring to Fig. 4, some signal values correspond to contributions from up to all four central symbol values. Hence, during the detection process, it eventually becomes impossible to distinguish between symbol values, where their distances based on the symbol detection scheme are very close. This mainly contributes to the saturation of the performance curve.

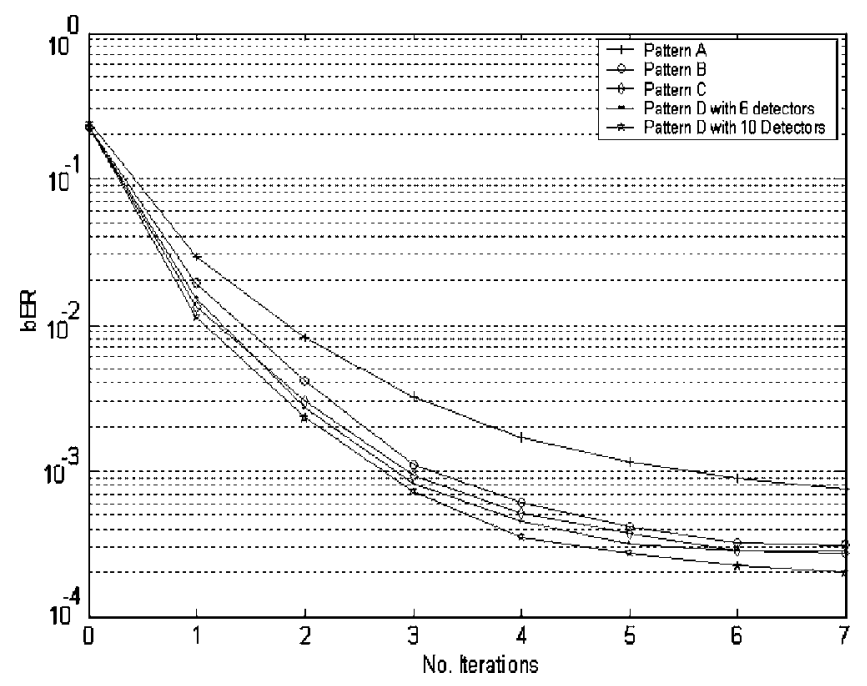

Fig. 11 Performance with varying iterations. 


\section{Conclusions}

We address the complexity restrictions imposed on symbol detection for multilevel TwoDOS using readout data generated by a linear channel model. We describe in detail the steps involved in developing a low complexity symbol detection scheme with acceptable performance for multilevel TwoDOS. The readout channel is simulated using a simple 2-D linear channel model based on the Braat-Hopkin's formula. The resulting signal levels, after the process of summation grouping to identify clusters with same overall pit area, are exploited to construct a symbol detection scheme that satisfies the optical storage performance requirements with relatively much lower complexity than a maximumlikelihood Viterbi-based algorithm. The symbol detection scheme is iterative, and we present four variations of the iterative algorithm based on how adjacent (vertically and horizontally) clusters exchange extrinsic information via a sliding windows operation. Results comparing the four configurations are presented in the presence of Gaussian noise. We also observe that performance improves with an increasing number of iterations, up to a certain point where the performance improvement between iterations is insignificant. The detector can be accompanied by a robust error correction scheme that will eliminate any remaining detection errors at the output of the symbol detector.

\section{Acknowledgments}

This work was a part of a "TwoDOS" project, funded by the European Commission with project number IST-200134168. All project members are acknowledged for their valuable contribution to this work.

\section{References}

1. W. M. J. Coene, "Two dimensional optical storage," Opt. Data Storage Conf. (ODS) Tech. Digest, Proc. SPIE 5069, pp. 90-92 (May 2003).

2. B. Stek, R. Otte, T. Jansen, and D. Modrie, "Advanced signal processing for the blu-ray disc system," Tech. Digest, ISOM/ODS, IEEE Electronic Library, pp. 263-265 (2002).

3. W. Weeks and R. E. Blahut, "The capacity and coding gain of certain checkerboard codes," IEEE Trans. Inf. Theory 44, 1193-1203 (1998).

4. T. Kato, S. Taira, T. Maeda, Y. Katayama, and T. Nishiya, "Twodimensional run length-limited code and partial response maximum likelihood system with multitrack recording," Intl. Symp. Opt. Mem. Opt. Data Storage, Tech. Digest, IEEE Electronic Library, pp. 51-53 (2002).

5. A. H. J. Immink, W. M. J. Coene, A. M. van der Lee, C. Busch, A. P. Hekstra, J. W. M. Bergmans, J. Riani, S. J. L. v. Beneden, and T. Conway, "Signal processing and coding for two-dimensional optical storage," GLOBECOM'03 7, 3904-3908 (Dec. 2003).

6. J. Braat, "Read-out of optical discs," in Principles of Optical Disc Systems, pp. 7-87, Adam Hilger Ltd, Bristol (1985).

7. H. Hieslmair, J. Stinebaugh, T. Wong, and M. O'Neill, "34 GB multilevel-enabled rewritable system using blue laser and high-NA optics," Joint Intl. Symp. Opt. Mem. Opt. Data Storage Tech. Dig., IEEE Electronic Library, pp. 7-9, (2002).

8. A. Moinian, L. Fagoonee, B. Honary, and W. M. J. Coene, "Linear channel model for multilevel two dimensional optical storage," in Proc. 7th Intl. Symp. Commun. Theory Applications, published by HW Communications Limited, pp. 352-356 (2003).

9. L. Fagoonee, A. Van Der Lee, A. Moinian, B. Honary, and W. Coene, "Experimental characterization for the binary two-dimensional optical storage channel," presented at IEEE Intl. Conf. Commun. (ICC4), Paris, France, 20-24 June 2004.

10. K. Kiyono, T. Hashizume, and M. Horie, "Application of multi-level recording with growth-dominant material to CD and blue DVR generations," Joint Intl. Symp. Opt. Mem. Opt. Data Storage Tech. Digest, IEEE Electronic Library, pp. 66-68 (2002).

11. H. Hieslmair, J. Stinebaugh, T. Wong, M. O'Neill, M. Kuijper, and G. Langereis, "34GB multilevel-enabled rewritable system using blue laser and high-numeric aperture optics," Jpn. J. Appl. Phys., Part 1 42(2B), 1074-1075 (Feb. 2003).
12. J. Pasman, "Vector theory of diffraction," in Principles of Optical Disc Systems, Chap. 3, pp. 88-124, E. R. Pike, Adam Hilger, Bristol, UK (1985)

13. W. M. J. Coene, "Nonlinear signal-processing model for scalar diffraction in optical recording," Appl. Opt. 42(32), pp. 6525-6535 (Nov. 2003).

14. S. G. Stan, "The CD-ROM drive: A brief system description," Chap. 2, Kluwer, Boston (1998).

15. E. Hetch, Optics, Addison-Wesley Publishing, Reading, MA (1987).

16. J. F. Heanue, K. Gurkan, and L. Hesselink, "Signal detection for page-access optical memories with intersymbol interference," Appl. Opt. 35(14), 2431-2438 (May 1996).

17. C. L. Miller, B. R. Hunt, M. A. Neifeild, and M. W. Marcellin, "Binary image reconstruction via 2-D Viterbi search," Proc. Intl. Conf. Image Process. 1, 181-184 (1997).

18. A. P. Hekstra, W. M. J. Coene, and A. H. J. Immink, "Refinements of multitrack Viterbi bit-detection," Accepted in publication: IEEE Trans. Magn., SPS-30, 2005.

19. T. Narahara, S. Kobayashi, M. Hattori, Y. Shimpuku, G. van den Enden, J. Kahlman, M. van Dijk, and R. van Woudenberg, "Optical disc system for digital video recording," Jpn. J. Appl. Phys., Part 1 39(2B), 912-919 (2000).

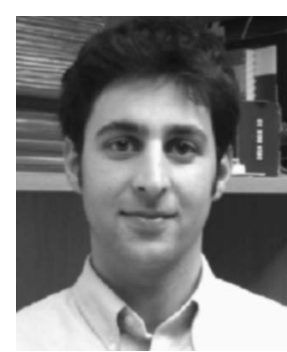

Abdolhosein Moinian received his BSc in electrical engineering from Sharif University of Technology (Iran), and MSc on mobile and personal radio communications from Lancaster University United Kingdom (UK) in 2000 and 2002, respectively. He is doing his $\mathrm{PhD}$ on signal processing and coding for optical storage, and has been a research associate at Lancaster University since 2002. His research interests include signal processing, error control coding, channel estimation and modeling, and wireless communications.

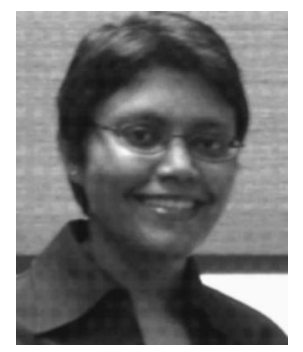

Lina Fagoonee received her BEng (hons) in electronic communications engineering and $\mathrm{PhD}$ in multifunctional turbo receiver design based on partial unit memory codes from Lancaster University in 1999 and 2003 , respectively. She was a research assistant from 1999, and was then offered a research associate (2002) position at Lancaster University to work on signal processing and coding for optical storage. She currently works as a lecturer in the Department of Communication Systems at Lancaster University. Her general research interests include error control coding, channel estimation and modeling, signal processing optical/magnetic storage, and wireless communications. She has published more than a dozen papers in channel estimation and modeling for 2-D optical storage, turbo and woven turbo codes based on partial unit memory codes, and signal processing in radio communication channels. She is a member of the IEE and IEEE, and is a student liaison officer of the IEEE chapter for information theory for the UK and Republic of Ireland.

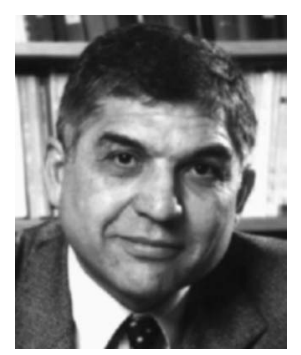

Bahram Honary received his MSc in digital communications and $\mathrm{PhD}$ in error protection techniques for bursty channels from the University of Canterbury (UK) in 1976 and 1982, respectively. In 1984 he was appointed a senior lectureship at Coventry Polytechnic. In 1988, he joined the Department of Engineering, University of Warwick. In 1992, he took up the position of chair of communications engineering at Lancaster University. His current research interests include channel coding and application to radio communication channels, secure communication applications, and modem design and synchronization. He is the chairman of the IEEE chapter for information theory for the UK and Republic of Ireland. He is a Fellow of the Institution of Electrical Engineering (IEE) and Fellow of the Institute of Mathematics and Applications (IMA). 